\title{
叶绿素降解产物的环丙基化及其二氢卟吩衍生物的合成
}

\author{
刘 洋 ${ }^{a}$ 徐希森 ${ }^{a}$ 李家柱 ${ }^{a}$ 殷军港 ${ }^{a}$ 祁彩霞 ${ }^{b}$ 王进军*, $a, b$ \\ ( 烟台大学化学化工学院 烟台 264005) \\ ( ${ }^{b}$ 山东省黄金工程技术研究中心(工业应用) 烟台 264005)
}

\begin{abstract}
摘要 以叶绿素降解产物脱镁叶绿酸-a 甲酯为起始原料, 利用其二氢卟吩大环上的活性反应区域, 沿着 $\mathrm{N}^{21}-\mathrm{N}^{23}$ 轴向进 行官能团修饰，通过 1,3-偶极环加成反应、Grignard 反应和外接环的结构改造，在周环上的不同位置上引进了三元环烷 基结构, 完成了一系列未见报道的叶绿素类二氢卟吩衍生物的合成, 其化学结构均经 UV, IR, ${ }^{1} \mathrm{H}$ NMR, MS 及元素分析 予以证实, 同时也讨论了环丙基的引进对二氢卟吩的理化性质所产生的各种影响, 并对部分新化合物的体外光动力活 性进行了测试.
\end{abstract}

关键词＼cjkstart叶绿素-a; 二氢卟吩; 化学修饰; 环丙基化; 合成

\section{Cyclopropylation of Chlorophyllous Degradation Products and Synthesis of Chlorin Derivatives}

\author{
Liu, Yang ${ }^{a} \quad \mathrm{Xu}$, Xisen $^{a} \quad \mathrm{Li}$ Jiazhu $^{a} \quad$ Yin, Jungang $^{a} \quad$ Qi, Caixia $^{b} \quad$ Wang, Jinjun $^{*, a, b}$ \\ ( ${ }^{a}$ College of Chemistry and Chemical Engineering, Yantai University, Yantai 264005) \\ ( ${ }^{b}$ Shandong Applied Research Centre of Gold Nanotechnology (Au-SDARC), Yantai 264005)
}

\begin{abstract}
Pheophorbide-a methyl ester, as a degraded product from chlorophyll-a, was used as a starting material, and the modifications for functional groups along $\mathrm{N}^{21}-\mathrm{N}^{23}$-axis were completed by making use of reactive regions. The tri-membered ring structures were introduced to the macrocyclic chromophore in different position by 1,3-polar cycloaddition, Grignard reaction and reconstructions for exocyclic ring. A series of unreported chlorins related to chlorophyll were synthesized and their chemical structures were characterized by UV, IR, ${ }^{1} \mathrm{H}$ NMR, MS techniques and elemental analysis. The different effects on the physical and chemical properties of chlorophyllous chlorins by introduction of cyclopropyl group were discussed and in vitro photodynamic activities of some new compounds were determined.
\end{abstract}

Keywords chlorophyll-a; chlorin; chemical modification; cyclopropylation; synthesis

叶绿素-a (Chl-a) 的降解产物是一类具有特定碳架 结构的四吡咯大环化合物, 在新型光动力治疗 (photodynamic therapy, 简称PDT)抗癌药物的合成研究中一直占 有相当重要的位置, 相应的定量构效关系(QSAR)研究 表明，叶绿素类二氢卟吩周环连带结构的所占空间、连 接位置、几何形状以及氧化还原电位均能对抗癌疗效产 生深刻影响, 特别是活性官能团的引入和大环分子水脂 溶性的调整是合成理想 PDT 药物的重要前期工作 ${ }^{[1 \sim 3]}$. 叶绿素降解产物的 A-B 子环端向和 C-D 子环端向分别 呈亲脂和亲水溶性, 适当地改变其水脂分配系数可以不 同程度地提升大环分子对肿瘤细胞的积聚能力, 人们通
过合成带有各种不同取代基团的环状四吡咯化合物，已 经篎选出许多可用于光动力治疗的新型抗癌药物 ${ }^{[4,5]}$. 与引进亲水性官能基团不同, 在卟啉色基上建立具有反 应活性的脂溶性烷基则相对较少. 基于前期的工作基 础 ${ }^{[6,7]}$, 本文以叶绿素-a (Chl-a)为研究对象, 通过不同的 化学反应, 在叶绿素二氢卟吩周环的相应位置上构建环 状空间的烷基碳链，在改善和提高大环分子脂溶性的同 时，也引进了具有良好反应活性并能进一步实施化学修 饰的环丙基结构, 合成出一系列未见报道的环丙基取代 叶绿素类二氢卟吩衍生物, 并对部分新化合物的光动力 抗癌活性进行了测试.

*E-mail: wjj1955@163.com

Received October 6, 2013; revised November 8, 2013; published online November 20, 2013.

Project supported by the National Natural Science Foundations of China (No. 21272048) and the Project of Shandong Applied Reaearch Centre of Gold Nanotechnology (2011).

国家自然科学基金(No. 21272048)和山东省黄金工程技术研究中心(2011 年度)资助项目. 


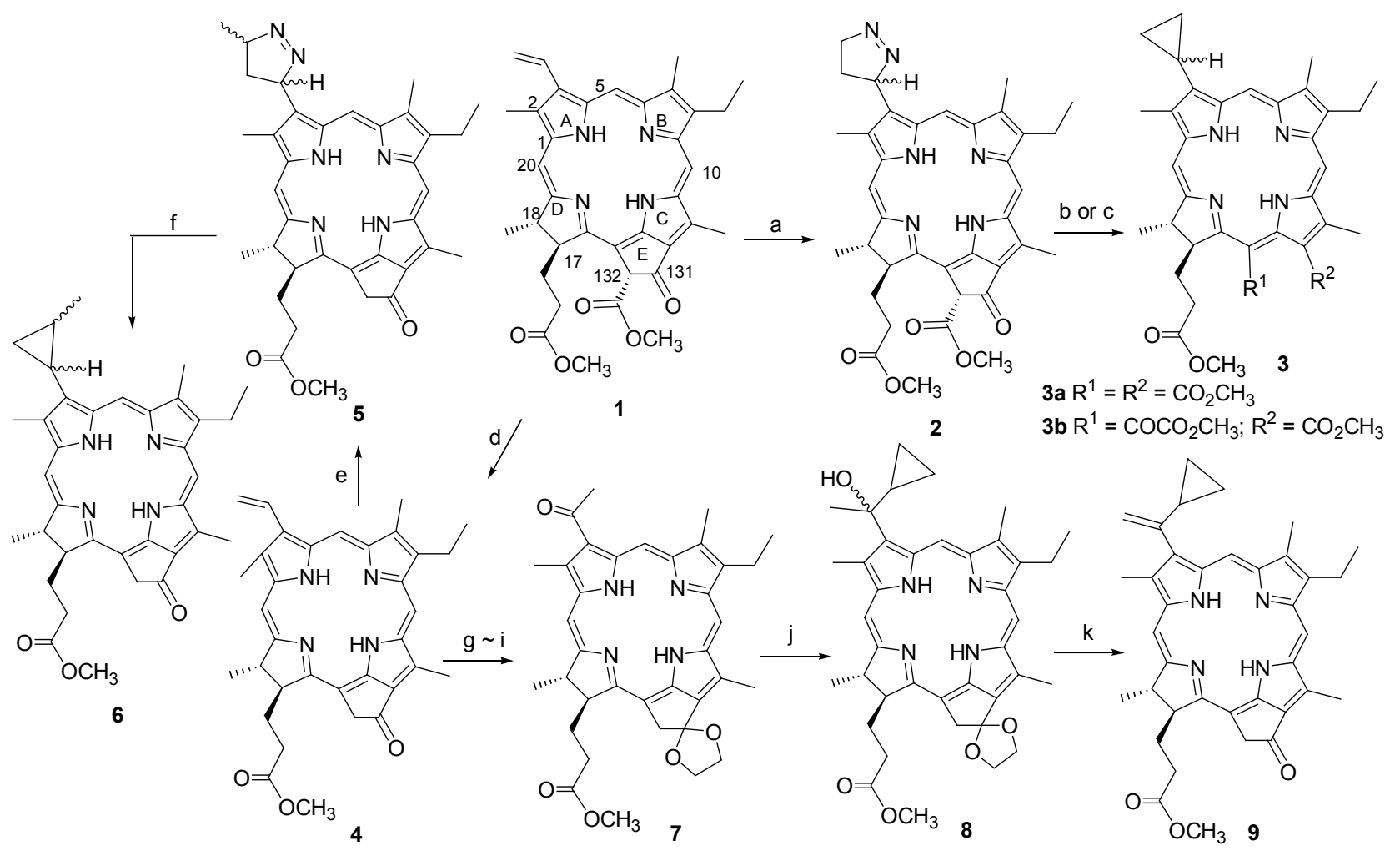

Reagents and conditions: (a) $\mathrm{CH}_{2} \mathrm{~N}_{2} / \mathrm{CH}_{2} \mathrm{Cl}_{2}$; (b) $\mathrm{KOH} / \mathrm{EtOH} / \mathrm{Pyr} / \mathrm{PhOPh}, 180^{\circ} \mathrm{C}$ for $3 \mathrm{a}$; (c) $\mathrm{KOH} / \mathrm{O}_{2} / \mathrm{C}_{3} \mathrm{H}_{7} \mathrm{OH} / \mathrm{PhOPh}, 180^{\circ} \mathrm{C}$ for $3 \mathbf{b}$; (d) $\mathrm{AcOH}, 110^{\circ} \mathrm{C}$; (e) $\mathrm{CH}_{3} \mathrm{CHN}_{2} / \mathrm{CH}_{2} \mathrm{Cl}_{2}$; (f) $\mathrm{PhOPh} / 180^{\circ} \mathrm{C}$; (g) $\mathrm{HBr} / \mathrm{AcOH}$; (h) $\left(\mathrm{CH}_{2} \mathrm{OH}\right)_{2} / \mathrm{TMSCl}$; (i) $\mathrm{Pr}_{4} \mathrm{NRuO}_{4} / \mathrm{N}$-Me morpholine $\mathrm{N}$-oxide; (j) $\left(\mathrm{CH}_{2}\right)_{2} \mathrm{CHMgBr} / \mathrm{THF} ;(\mathrm{k}) \mathrm{TsOH} / \mathrm{Benzene}$

\section{Scheme 1}

脱镁叶绿酸-a 甲酯 $(1, \mathrm{MPa})$ 经重氮甲烷处理后, 所 得 3-吡唑啉基取代的焦脱镁叶绿酸-a 甲酯(2)不经分离, 在碱性条件下分别进行 E-环重排和空气氧化反应, 并 在二苯醚中直接实施 $\mathrm{C}(3)$-吡唑啉基的热裂解, 分别得 到 31\%的 3-环丙基-3-去乙烯基二氢卟吩- $p_{6}$ 三甲酯(3a) 和 42\%的 3-去乙烯基红紫素-7-三甲酯(3b). 选用重氮乙 烷用相同的方法处理焦脱镁叶绿酸-a 甲酯 $(4, \mathrm{MPPa})$, 得 到中等产率的 3-(5-甲基吡唑啉基)焦脱镁叶绿酸-a 甲酯 (5), 其高温裂解在 3-位上同样构成三元环结构, 以 70\% 的产率分离出 3-位甲基环丙基取代的同系物 6. 从 MPPa (4)开始, 经过 3-位双键的亲电加成、E-环宸基保 护和所形成差基的氧化反应, 以 38\%的产率得到 3-乙酰 基焦脱镁叶绿酸-a 甲酯(7), 与环丙基溴化镁的 Grignard 反应给出二氢卟吩酩醇 $(\mathbf{8}, 56 \%)$, 酸性条件下的脱水反 应给出 3a-环丙基焦脱镁叶绿酸-a 甲酯(9,64\%).

利用 MPPa (4)的 C(3)-乙烯基与多聚甲醛和 $N$-甲基 甘氨酸进行 1,3-偶极环加成反应, 试图在 3-位上构建氢 化吡咯环系, 结果没有得到预期产物, 但从复杂的混合 物中, 意外地分离出 $13^{2}$-位带有螺环结构的焦脱镁叶绿 酸-a 甲酯 $(\mathbf{1 0}, 11 \%)$. 用重氮甲烷处理 4 并直接在二苯醚 中完成热裂解，一步法可以相对高产率地得到 3-环丙基 焦脱镁叶绿酸-a 甲酯(11，67\%)，继续与过氧化氢/稀盐 酸进行亲电取代反应，以理想的产率给出 20 -氯焦脱镁 叶绿酸-a 甲酯(12,74\%); 化合物 $\mathbf{1 1}$ 的空气氧化反应得 到 $23 \%$ 的二氢卟吩二酮(13), 进一步用氢氧化钠甲醇溶 液处理，以 $80 \%$ 的产率转化成 3-环丙基红紫素-18 甲酯 (14), 再与环丙甲胺在甲苯中共回流, 得到 $61 \%$ 的双环 丙基取代的红紫素-18 亚酰胺(15).

\section{1 结果与讨论}

\section{1 三元碳环的引入及其对二氢卟吩理化性质所产生 的影响}

环丙基除了环烷烃所具有的良好脂溶性以外，其独 有的价键结构形成较强的环张力，并赋予给环丙基有别 于其他脂环碳络的特殊性质, 因此, 在二氢卟吩周环上 构建三元碳环结构, 既能改善四吡咯大环的物理性质, 也为后续的结构修饰建立了潜在的切入位点. 吡唑啉五 元杂环的热裂解反应是构建环丙基碳架最为便利的方 法, 作为 1,3-偶极环加成的亲偶极体, 叶绿素降解产物 的 C(3)-位乙烯基提供了有效反应区域，与重氮烷烃偶 极体可以顺利地完成环合反应, 在周环上形成了吡夾啉 或者烷基取代吡唑啉环系结构, 进而热裂解成相应的三 


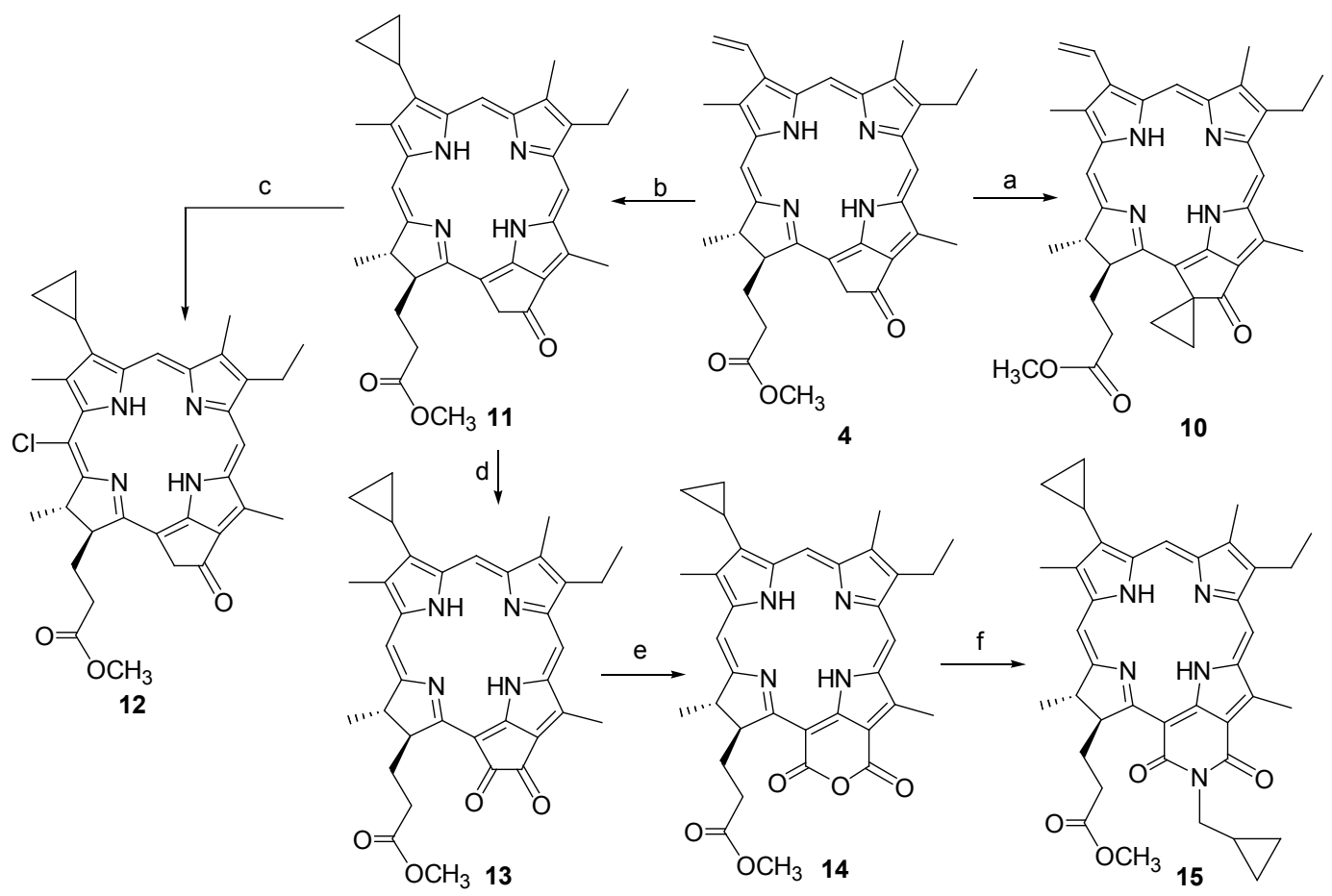

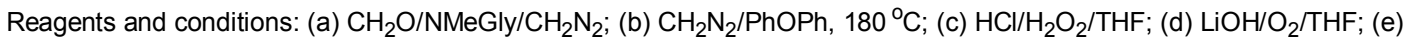
$\mathrm{KOH} / \mathrm{EtOH} / \mathrm{O}_{2}$; (f) $\mathrm{NH}_{2} \mathrm{CH}_{2} \mathrm{CH}\left(\mathrm{CH}_{2}\right)_{2}$

\section{Scheme 2}

元碳环.

重氮烷烃具有三原子四电子的 $\pi$ 体系, 其分子轨道 类似于烯丙基负离子, 其最高占有轨道(HOMO)的对称 性和普通的共轭双烯相同, 因此, 1,3-偶极环加成和 Diels-Alder 反应的机理相近, 是热允许的同面环加成 $\left[\pi^{4} \mathrm{~S}+\pi^{2} \mathrm{~S}\right]$ 过程. 焦脱镁叶绿酸-a 4 的 3-位乙烯基通过色 基中的二氮杂-18-轮烯与 $\mathrm{E}$-环羰基形成远程共轭, 由于 E-环羰基的插烯作用和电子效应，促成 3a-和 3b-位碳原 子上分别带有部分负电荷和正电荷, 因而与重氮烷基偶 极体的环合取向采取反马氏加成的方式(途径 a), 在 3位上形成吡唑啉环 A (B); 如果偶极体以马氏加成的方 式(途径 b)与 $C(3)$-乙烯基进行加成的话，应该环合成同 分异构的五元杂环 $\mathbf{A}_{\mathbf{1}}\left(\mathbf{B}_{1}\right)$. 吡唑啉基的热裂解可以经 由无氢迁移的断裂方式 $\left(\mathbf{A}\right.$ 和 $\left.\mathbf{A}_{\mathbf{1}}\right)$ 形成三元环结构 $\mathbf{C}$, 也 能够通过有氢键迁移的断裂方式 $\left(\mathbf{B}\right.$ 和 $\left.\mathbf{B}_{1}\right)$ 构成环外烯键 (D 和 $\mathbf{D}_{1}$ ). 由于在 $\mathbf{B}$ 的热裂解过程中迁移的氢原子活性 较弱, 所形成的双键与大环色基也没有形成共轭, 很难 以这种方式完成重排; 马氏加成产物 $\mathbf{B}_{1}$ 则正好相反, 3a位氢原子类似于苠基位质子，所以， $\mathbf{D}_{1}$ 应该作为主要的 裂解产物. 然而, 所有吡唑啉环的热裂解反应均给出环 丙基取代的二氢卟吩, 在反应中尚未发现具有端烯结构 的重排产物 $\mathrm{D}_{1}$, 其结果证明(焦)脱镁叶绿酸是经历途径 $\mathrm{a}$ 完成与重氮烷烃的 1,3-偶极环加成, 而不是按照途径 $\mathrm{b}$ 的方式进行相应的反应(图 1).
在 MPPa (4)的另一偶极环加成的设计中, 多聚甲醛 和 $N$-甲基甘氨酸在甲苯中的回流并没有形成亚甲化腈 类 1,3-偶极体 $\mathbf{E}$ ，因此没有发生相应的环加成反应. 根 据化合物 10 中 E-环所连带的三元螺环结构, 可以确定 甲醛与外接环酮发生了羟醛缩合, 在 $13^{2}$-位上形成了对 偶极体具有更强亲合性的亚甲基，因此，烯酮 $\mathbf{F}$ 与重氮 甲烷的反应没有形成化合物 $\mathbf{A}$, 即未在 3-位双键上发生 环加成反应，而与具有更强亲偶极的 $13^{2}$-位亚甲基相互 作用，以螺环连接的形式构成了吡唑啉基，进而裂解成 具有螺环碳架的二氢卟吩 10. 除此之外, 利用二氢卟吩 周环上的活性反应基团，选择带有环丙基结构的反应试 剂与之进行化学反应，也是建立三元环烷结构的有效方 法, 环丙基溴化镁与 3-乙酰基焦脱镁叶绿酸-a 衍生物 7 的格氏反应，顺利地将环丙基连接在 3a-位上; 红紫素18 的外接环酩的胺解反应是修饰叶绿素降解产物的常 用方法, 采用环丙基甲胺与 3-环丙基红紫素-18 的缩合 反应，也在外接环的酰亚胺氮原子上引进了环丙烷结 构.

叶绿素类二氢卟吩的 PDT 活性与大环分子的水脂 溶性关联甚密，调整 PDT 用光敏剂的分子极性也是改 善其光动力疗效的重要手段. 与所有叶绿素-a 的降解产 物相比, C(3)-环丙基取代的二氢卟吩衍生物的 $R_{\mathrm{f}}$ 值相对 较高，说明乙烯基向环丙基的转化可以提高 $\mathrm{C}-\mathrm{D}$ 环端向 的脂溶性，从而改变了大环分子的极性. 在其他位置连 


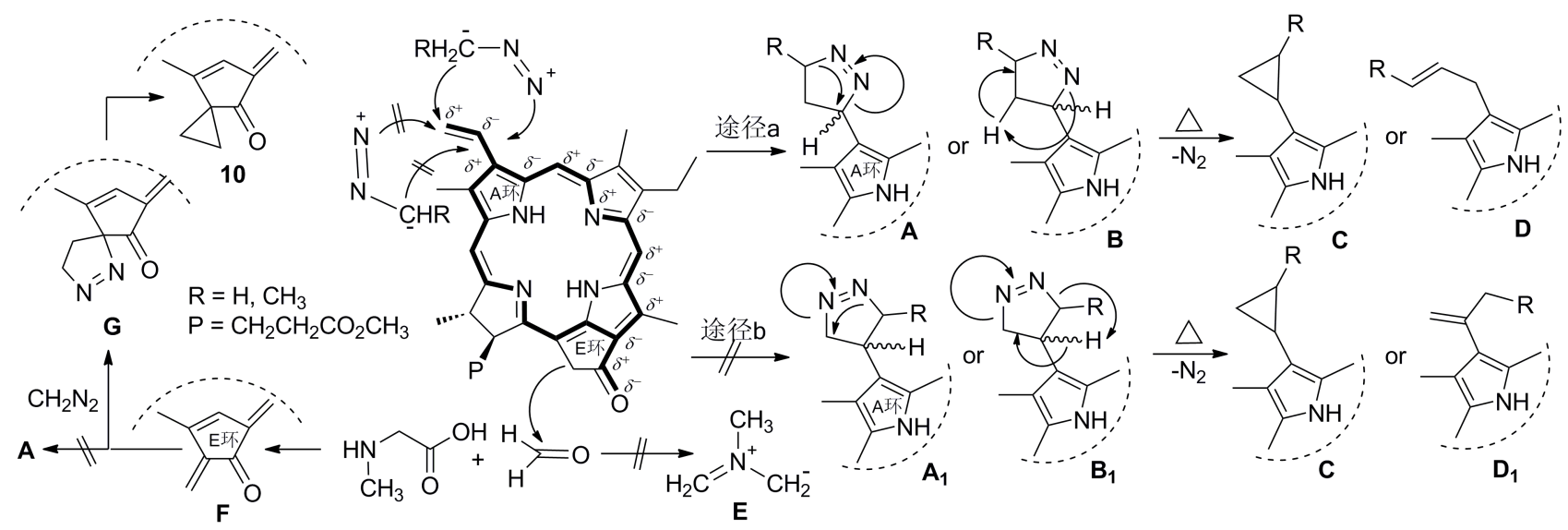

图 1 叶绿素衍生物的 1,3-偶极环加成与三元环烃结构的形成

Figure 2 The 1,3-dipolar cycloations of chlorophyllous derivatives and the formation of tricyclic alkane structure

接环丙基结构，同样也促成了极性的降低，例如，8 或 9 的 3a-位以及红紫素-18 亚酰胺的外接环氮原子上三元 碳环的引入, 均不同程度地改变了二氢卟吩的分子极 性.

紫外-可见光谱是叶绿素类二氢卟吩在光医学等诸 多应用方面所要考量的重要参数, 在不同位置上引进环 丙基, 可以不同程度地改变大环分子的光物理性质, 特 别是沿着 $\mathrm{N}^{21}-\mathrm{N}^{23}$ 轴向的结构修饰对分子的最大可见光 吸收(Qy 吸收)能够产生强烈的影响 ${ }^{[7]}$. 当 3-位乙烯基转 化成环丙基以后, 由于失去了环外碳碳双键与大环色基 的共轭体系, 所有叶绿素衍生物的 Qy 谱带均形成 $6 \sim 8$ $\mathrm{nm}$ 左右的蓝移; 尽管化合物 9 在 3-位上仍然连有乙烯 基, 但由于 $3 \mathrm{a}$-位环丙基团的引进, 与 2-位甲基和 5-位$m e s o$-氢原子形成空间排斥, 在一定程度上破坏了环外 烯键与四吡咯母环的共平面结构, 从而促使 9 的最大可 见光吸收比 $\mathrm{MPPa}(4)(\mathrm{Qy}=666 \mathrm{~nm})$ 蓝移了 $5 \mathrm{~nm}$ (图 2 中 $\mathrm{A}$ 式 $)^{[8]}$. 另一方面, 3-位环丙基和螺连于外接 E-环上的 的环丙烷结构可以通过其特有的化学键参与共轭. 例 如, 当 3-位乙烯基还原成乙基时, 所得二氢卟吩的最大 可见光吸收波长蓝移至 $654 \mathrm{~nm}^{[9]}$, 而环丙基连接于色基 上的化合物 11 的相应吸收峰却落在 $658 \mathrm{~nm}$ 处, 说明 3位环丙基的弯曲 $\sigma$ 键与大环 $\pi$-体系能够形成一定程度的 电子离域(图 2 中 $\mathbf{B}$ 式), 因而比 3-乙基焦脱镁叶绿酸的 Qy 吸收稍微红移; 与化合物 $\mathbf{4}$ 相比, 在 E-环上含有螺连
三元碳环结构的化合物 $\mathbf{1 0}$ 的 Qy 峰值同样红移了 $4 \mathrm{~nm}$. 虽然外接环上的环丙基距离大环 $\pi$-体系相对较远，但是 其特殊的 $\sigma$ 键仍然可以与 E-环羰基形成同共轭关系(图 2 中 $\mathbf{C}$ 式), 进而促成了大环分子的最大可见光的吸收波 长向长波方向推移.

虽然乙烯基和环丙基都具有多电子性而容易发生 加成反应，但 3-位乙烯基转化为环丙基后，叶绿素类二 氢卟吩的亲电反应位点完全集中在 20-meso-位, 3-位环 丙基却表现出一定的稳定性，在各种不同的反应条件 下, C(3)-环丙基均保持不变; 在与甲醛和重氮甲烷发生 一系列的化学反应之后, 化合物 10 作为最为稳定的产 物从反应体系中分离出来. 说明其外接 E-环中的螺连 三元环结构也相对稳定. 因此, 选择合适的化学试剂和 反应方法, 是对叶绿素周环上的环丙烷结构实施进一步 修饰的关键.

\section{2 环丙基取代二氢卟吩衍生物的体外光动力药理活} 性

叶绿素类二氢卟吩的光动力活性与其周环上的化 学结构密切相关, 特别是连接在 $\mathrm{N}^{21}-\mathrm{N}^{23}$-轴向上的取代 基团，可以通过自身所特有的空间效应和电子效应对四 吡咯大环分子的 PDT 抗癌疗效产生不同程度的影响. 考虑到 3-位环丙基结构的特殊性，分别选择 2, 3a, 3b, 11,12 和 15 以及焦脱镁叶绿酸-a 甲酯(4)为待测化合物,
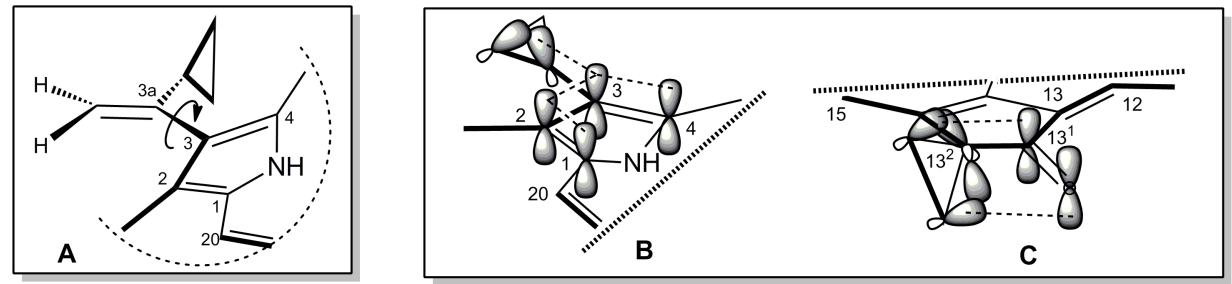

图 2 二氢卟吩周环上三元碳环的取代基效应及其对紫外-可见光谱的影响

Figure 2 The substituent effects of tri-carbocycles on the periphery of chlorins and the effection on their UV-Vis spectra 
以临床光动力抗癌药物光敏素(Photofrin)和中位 meso四羟苯基二氢卟吩 (mTHPC) 为对照品, 在光照条件下, 采用 MTT 比色法对上述化合物对小鼠肉瘤 S-180 细胞 的体外光动力活性进行测定. 以所测化合物浓度的对数 为因变量, 以细胞抑制率为应变量作求得半抑制率 $\left(\mathrm{IC}_{50}\right)$. 从表 1 可以看出, 所有测试化合物对小鼠肉瘤 S-180 细胞都具有较强的光动力活性, 其 $\mathrm{IC}_{50}$ 值均低于 $0.400 \mu \mathrm{mol} / \mathrm{L}$, 对病毒细胞的抑制能力远高于第一代临 床的光动力抗癌药物 Photofrin; 尽管化合物 3a, 3b, 11, 12,15 的 PDT 活性低于对照药物目前临床应用效果最好 的 PDT 药物 mTHPC, 但均高于作为叶绿素最初降解产 物的焦脱镁叶绿酸-a 甲酯(4), 而3-位连有五元杂环的二 氢卟吩 2 的相应抑制效力甚至高于 mTHPC 2.4 倍.

当化合物 4 的 3-位乙烯基转化成环丙基以后, 所形 成的大环分子 11 对小鼠肉瘤 S-180 细胞的抑制率增强, 而 20-位氯原子取代的二氢卟吩 12 则大幅度地提高了 PDT 活性. 叶绿素类二氢卟吩的外接环修饰也使其 $\mathrm{IC}_{50}$ 值呈示出规律性的变化, 具有开链结构的二氢卟吩 $-\mathrm{p}_{6}$ 三甲酯(3a)和红紫素-7 三甲酯(3b)所表现的较高的光动 力活性, 而具有外接环结构的焦脱镁叶绿酸(4)和红紫 素-18 (15)的半抑制率 $\mathrm{IC}_{50}$ 则相对较低. 对照 C-D 环端 向的化学结构可以发现, 所连接的取代基团的极性越 高, 其光动力抗癌活性越强, 在 13-位和 15-位上分别连 有甲氧酰基和甲氧乙二酰基的 $\mathbf{3 b}$ 的 $\mathrm{IC}_{50}$ 值仅为 0.124 , 而具有最小极性的红紫素-18 衍生物 15 的相应测定值则 达到 0.268 .

\section{2 结论}

利用叶绿素类二氢卟吩芳香性大环上的活性反应 区域, 通过环上烯键与重氮烷烃的 1,3-偶极环加成, 或 者选用含环丙基亲核试剂直接参与反应, 在周环 $\mathrm{N}^{21}-\mathrm{N}^{23}$ 轴向的不同位置上构建和引进了三元环烷结构. 三元环碳架可以有效地改善大环分子的水脂溶性, 并促 成其电子光谱产生规律性变化, 同时在四吡咯环上形成 了新的反应区域, 为进一步改变叶绿素降解产物的化学 结构, 完善其光物理、光生物等诸多性质提供了新的切 入途径.

\section{3 实验部分}

\section{1 仪器与试剂}

元素分析用 Perkin-Elmer 2400 型元素分析仪测定;
IR 用 Perkin-Elmer 1730 型红外分光光度仪测定 $(\mathrm{KBr}$ 压 片); UV-Vis 用 UV-160A 型紫外分光光度计测定; ${ }^{1} \mathrm{H}$ NMR 用 Brucker ARX-300 型核磁共振仪测定, 内标为 TMS; ESI-MS 用 Bruker Daltonics Esquire3000 plus 或者 Varian 500-MS IT 质谱仪测定; 所用试剂均为分析 纯或化学纯, 脱镁叶绿酸-a 甲酯(1)和焦脱镁叶绿酸-a (4)按文献[10]制备；3-乙酰基-13 ${ }^{1}$-(1.4-二氧环戊基)-3-去 乙烯基-焦脱镁叶绿酸甲酯-a (7)按文献[11]制备.

\subsection{3 -环丙基-3-去乙烯基二氢卟吩- $\mathrm{p}_{6}$ 三甲酯(3a)的合 成}

$128 \mathrm{mg}(0.211 \mathrm{mmol})$ 溶解于 $2 \mathrm{~mL}$ 二氯甲烷的脱镁 叶绿酸-a 甲酯(1)加入到 $15 \mathrm{~mL}$ 二氯甲烷中, 加入 $25 \mathrm{~mL}$ 自制的重氮甲烷乙醚溶液，室温下避光密封放置 $3 \mathrm{~d}$ 后， 摚拌下加入 $5 \mathrm{~mL}$ 乙酸, 再加入 $30 \mathrm{~mL}$ 水分层, 有机相用 水洗涤 $(25 \mathrm{~mL} \times 3)$, 减压浓缩至干. 将所得黑色粘稠物 重新溶解于 $10 \mathrm{~mL}$ THF 中, 加入 $5 \mathrm{~mL} 25 \%$ 的氢氧化锂 水溶液, 室温搅拌 $4 \mathrm{~h}$, 用乙酸将 $\mathrm{pH}$ 值调至 3 , 分出有机 层并浓缩至干，先后加入 $10 \mathrm{~mL}$ 吡啶和 $15 \mathrm{~mL} 25 \%$ $(m / V)$ 的氢氧化钾乙醇溶液, 氮气保护, 室温搅拌 $1 \mathrm{~h}$ 后 迅速回流 $1.5 \mathrm{~h}$ ，再向反应体系加入 $60 \mathrm{~mL}$ 水，用 $10 \%$ 的 硫酸调制 $\mathrm{pH}$ 至 3 , 用二氯甲烷萃取 $(25 \mathrm{~mL} \times 2)$, 无水硫 酸钠干燥合并有机相, 除去大部分溶剂, 经重氮甲烷甲 基化, 浓缩后加入 $5 \mathrm{~mL}$ 二苯醚, $160{ }^{\circ} \mathrm{C}$ 下搅拌 $1.5 \mathrm{~h}$, 加 入正己烷稀释，过滤除去大部分二苯醚，浓缩后经硅胶 柱层析分离[洗脱剂: $V$ (石油醚) $: V$ ( 乙酸乙酯 $)=4: 1$ ], 得 $42 \mathrm{mg}$ 暗红色固体产物 3a $(0.065 \mathrm{mmol})$, 产率 31\%. m.p. 199 201 ${ }^{\circ} \mathrm{C}$; UV-Vis $\left(\mathrm{CH}_{2} \mathrm{Cl}_{2}\right) \lambda_{\max }\left[\varepsilon /\left({\mathrm{L} \cdot \mathrm{mol}^{-1}}^{-1}\right.\right.$ $\left.\mathrm{cm}^{-1}\right)$ ]: $398\left(1.32 \times 10^{5}\right), 496\left(1.19 \times 10^{4}\right), 527$ $\left(5.28 \times 10^{3}\right), 605\left(5.28 \times 10^{3}\right), 658\left(3.96 \times 10^{4}\right) \mathrm{nm} ;{ }^{1} \mathrm{H}$ NMR $\left(\mathrm{CDCl}_{3}\right) \delta:-0.91$ (br s, $\left.1 \mathrm{H}, \mathrm{NH}\right), 0.56($ br s, $1 \mathrm{H}$, $\mathrm{NH}), 1.43 \sim 1.55(\mathrm{~m}, 2 \mathrm{H}, 3 \mathrm{~b}-\mathrm{H}), 1.30 \sim 1.38(\mathrm{~m}, 2 \mathrm{H}$, $3 \mathrm{~b}-\mathrm{H}), 1.68$ (t, $\left.J=7.5 \mathrm{~Hz}, 3 \mathrm{H}, 8-\mathrm{CH}_{3}\right), 1.84$ (d, $J=7.1 \mathrm{~Hz}$, $\left.3 \mathrm{H}, 18-\mathrm{CH}_{3}\right), 1.98 \sim 2.08(\mathrm{~m}, 1 \mathrm{H}, 17 \mathrm{a}+17 \mathrm{~b}-\mathrm{H}), 2.16 \sim$ $2.24(\mathrm{~m}, 2 \mathrm{H}, 17 \mathrm{a}+17 \mathrm{~b}-\mathrm{H}), 2.30 \sim 2.42(\mathrm{~m}, 1 \mathrm{H}, 17 \mathrm{a}+$ $17 \mathrm{~b}-\mathrm{H}), 2.70 \sim 2.78$ (m, 1H, 3a-H), 3.72 (q, $J=7.6 \mathrm{~Hz}, 2 \mathrm{H}$, $8 \mathrm{a}-\mathrm{H}$ ), 3.26, 3.31, 3.51, 362, 4.16, 4.21 (each s, each 3H, $\left.\mathrm{OCH}_{3}+\mathrm{CH}_{3}\right), 4.36(\mathrm{q}, J=7.0 \mathrm{~Hz}, 1 \mathrm{H}, 18-\mathrm{H}), 5.16(\mathrm{~d}, J=$ $8.6 \mathrm{~Hz}, 1 \mathrm{H}, 17-\mathrm{H}$ ), 8.54, 9.55, 9.64 (each s, each $1 \mathrm{H}$, meso-H); IR (KBr) v: $3449(\mathrm{~N}-\mathrm{H}), 2956(\mathrm{C}-\mathrm{H}), 1740 \sim$ $1701(\mathrm{C}=\mathrm{O}), 1654(\mathrm{C}=\mathrm{C}), 1560$ (chlorin skeleton), 1458,

表 1 部分叶绿素类二氢卟吩对小鼠肉瘤-180 细胞的半抑制率

Table $1 \mathrm{IC}_{50}$ results of chlorophyllous chlorins on mouse sarcoma S-180 cell line

\begin{tabular}{cccccccccc}
\hline Compd. & Photofrin & mTHPC & $\mathbf{2}$ & 3a & 3b & $\mathbf{4}$ & $\mathbf{1 1}$ & $\mathbf{1 2}$ & $\mathbf{1 5}$ \\
\hline $\mathrm{IC}_{50} /\left(\mu \mathrm{mol} \cdot \mathrm{L}^{-1}\right)$ & 0.736 & 0.057 & 0.025 & 0.171 & 0.124 & 0.363 & 0.192 & 0.098 & 0.268 \\
\hline
\end{tabular}


1400, 1073, $669 \mathrm{~cm}^{-1}$; MS m/z: $639.3(\mathrm{M}+\mathrm{H})^{+}$. Anal. calcd for $\mathrm{C}_{37} \mathrm{H}_{42} \mathrm{~N}_{4} \mathrm{O}_{6}$ : C 69.57, H 6.63, N 8.77; found $\mathrm{C}$ 69.69, H 6.55, N 8.99.

\subsection{3-环丙基-3-去乙烯基红紫素-7 三甲酯(3b)的合成}

将 $111 \mathrm{mg}$ 脱镁叶绿酸甲酯 $(\mathbf{1})(0.183 \mathrm{mmol})$ 溶解于 $10 \mathrm{~mL}$ 二氯甲烷中, 加入 $25 \mathrm{~mL}$ 自制的重氮甲烷乙梄溶 液, 室温下避光密封放置 $3 \mathrm{~d}$, 搅拌下加入 $5 \mathrm{~mL}$ 乙酸, 再加入二氯甲烷和 $30 \mathrm{~mL}$ 水分层, 有机相用水洗涤 $(25$ $\mathrm{mL} \times 3$ ) 分出有机层, 用无水硫酸钠干燥后减压浓缩至 干, 先后加入 $100 \mathrm{~mL}$ 乙醚、含有 $12 \mathrm{~g}$ 氢氧化钾的 $15 \mathrm{~mL}$ 正丙醇和 $15 \mathrm{~mL}$ 吡啶, 通入空气剧烈搅拌 $2 \mathrm{~h}$ 后, 用水 萃取反应混合物至醚层无绿色(每次 $35 \mathrm{~mL}$ ), 合并水相, 以 $25 \%$ 的硫酸调节 $\mathrm{pH}$ 值为 $2 \sim 3$, 再用二氯甲烷萃取至 水层无绿褐色, 立即用重氮甲烷处理有机相, $10 \mathrm{~min}$ 后 减压除尽溶液, 加入 $10 \mathrm{~mL}$ 二苯醚将所得粘稠物溶解, 并于 $160{ }^{\circ} \mathrm{C}$ 下搅拌 $1.5 \mathrm{~h}$, 加入正已烷稀释, 过滤除去大 部分二苯醚, 浓缩后经硅胶柱层析分离[洗脱剂: $V($ 石油 醚) $: V($ 乙酸乙酯 $)=3: 1]$, 得 $51 \mathrm{mg}$ 暗红色固体产物 $\mathbf{3 b}$ (0.077 mmol), 产率 42\%. m.p. $213 \sim 215{ }^{\circ} \mathrm{C}$; UV-Vis

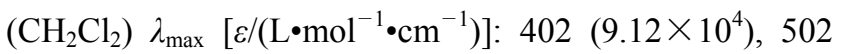
$\left(8.21 \times 10^{3}\right), \quad 540 \quad\left(7.30 \times 10^{3}\right), \quad 627 \quad\left(5.47 \times 10^{3}\right), \quad 658$ $\left(2.28 \times 10^{4}\right) \mathrm{nm} ;{ }^{1} \mathrm{H}$ NMR $\left(\mathrm{CDCl}_{3}\right) \delta: 0.03($ br s, $1 \mathrm{H}, \mathrm{NH})$, 0.05 (br s, $1 \mathrm{H}, \mathrm{NH}$ ), $1.48 \sim 1.56(\mathrm{~m}, 2 \mathrm{H}, 3 \mathrm{~b}-\mathrm{H}), 1.28 \sim 1.31$ (m, 2H, 3b-H), 1.65 (t, J=7.5 Hz, 3H, 8- $\left.\mathrm{CH}_{3}\right), 1.79$ (d, $\left.J=7.3 \mathrm{~Hz}, 3 \mathrm{H}, 18-\mathrm{CH}_{3}\right), 2.30 \sim 2.41(\mathrm{~m}, 2 \mathrm{H}, 17 \mathrm{a}+$ 17b-H), $2.02 \sim 2.18(\mathrm{~m}, 2 \mathrm{H}, 17 \mathrm{a}+17 \mathrm{~b}-\mathrm{H}), 2.67 \sim 2.76(\mathrm{~m}$, $1 \mathrm{H}, 3 \mathrm{a}-\mathrm{H}), 3.63$ (q, $J=7.5 \mathrm{~Hz}, 2 \mathrm{H}, 8 \mathrm{a}-\mathrm{H}), 3.16,3.27,3.53$, 3.61, 3.87, 4.13 (each s, each $3 \mathrm{H}, \mathrm{OCH}_{3}+\mathrm{CH}_{3}$ ), 4.26 (q, $J=7.2 \mathrm{~Hz}, 1 \mathrm{H}, 18-\mathrm{H}$ ), 4.67 (d, $J=7.5 \mathrm{~Hz}, 1 \mathrm{H}, 17-\mathrm{H}), 8.36$, 9.37, 9.52 (each s, each $1 \mathrm{H}$, meso-H); IR (KBr) v: 3451 $(\mathrm{N}-\mathrm{H}), 2925(\mathrm{C}-\mathrm{H}), 1737 \sim 1700(\mathrm{C}=\mathrm{O}), 1616(\mathrm{C}=\mathrm{C})$, 1548 (chlorin skeleton), 1460, 1223, 1034, 906, $746 \mathrm{~cm}^{-1}$; MS $m / z$ : $667.2(\mathrm{M}+\mathrm{H})^{+}$. Anal. calcd for $\mathrm{C}_{38} \mathrm{H}_{42} \mathrm{~N}_{4} \mathrm{O}_{7}: \mathrm{C}$ 68.45, H 6.35, N 8.40; found C 68.61, H 6.50, N 8.31.

\subsection{3 - $\left[\left(3^{\prime}(R, S)\right.\right.$-[1'-(4'(R,S)-甲基-吡唑啉基)]-3-去乙烯 基焦脱镁叶绿酸-a 甲酯(5)的合成}

在 $5 \mathrm{~mL}$ 干燥的四氢呋喃中溶解 $146 \mathrm{mg}(0.266$ $\mathrm{mmol}$ )焦脱镁叶绿酸-a 甲酯(4), 然后加入 $25 \mathrm{~mL}$ 重氮乙 烷乙醚溶液, 塞紧反应瓶口, 室温避光条件下放置 $18 \mathrm{~h}$, 常压下将未反应的重氮乙烷蒸馏回收, 再先后加入 25 $\mathrm{mL}$ 二氯甲烷和 $30 \mathrm{~mL}$ 水, 分出有机层, 水层用二氯甲 烷萃取 $(15 \mathrm{~mL} \times 3)$, 合并有机层, 用无水硫酸钠干燥, 减压浓缩, 所得剩余物经柱层析纯化 [洗脱剂: $V$ (石油 醚) : $V$ (乙酸乙酯) $=5: 2$ ], 得 $93 \mathrm{mg}$ 黑绿色固体 $\mathbf{5}$ $(0.154 \mathrm{mmol})$, 产率为 $58 \%$. m.p. $250 \sim 253{ }^{\circ} \mathrm{C}$; UV-Vis

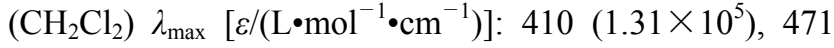
$\left(5.24 \times 10^{3}\right), \quad 504\left(1.13 \times 10^{4}\right), \quad 536\left(1.18 \times 10^{4}\right), \quad 607$ $\left(9.17 \times 10^{3}\right), 664\left(6.55 \times 10^{4}\right) \mathrm{nm} ;{ }^{1} \mathrm{H} \mathrm{NMR}\left(\mathrm{CDCl}_{3}\right) \delta:-$ $1.78(\mathrm{br}, 1 \mathrm{H}, \mathrm{NH}), 0.50(\mathrm{br}, 1 \mathrm{H}, \mathrm{NH}), 1.67$ (t, $J=7.6 \mathrm{~Hz}$, $\left.3 \mathrm{H}, 8 \mathrm{~b}-\mathrm{CH}_{3}\right), 1.71\left(\mathrm{~d}, J=7.1 \mathrm{~Hz}, 3 \mathrm{H}, 18-\mathrm{CH}_{3}\right), 1.81(\mathrm{~d}$, $\left.J=7.3 \mathrm{~Hz}, 3 \mathrm{H}, 4^{\prime}-\mathrm{CH}_{3}\right), 2.03(\mathrm{dd}, J=7.0,3.0 \mathrm{~Hz}, 1 \mathrm{H}$, $\left.3^{\prime}-\mathrm{H}\right), 2.23 \sim 2.39$ (m, 2H, 17a-H+17b-H), $2.31 \sim 2.37$ (m, $\left.1 \mathrm{H}, \quad 4{ }^{\prime}-\mathrm{H}\right), 2.51 \sim 2.74(\mathrm{~m}, 1 \mathrm{H}, 17 \mathrm{a}-\mathrm{H}+17 \mathrm{~b}-\mathrm{H}), 3.18$ (3.16), 3.30 (3.26), 3.61, 3.64 (each s, each $3 \mathrm{H}, \mathrm{CH}_{3}+$ $\mathrm{OCH}_{3}$ ), 3.67 (q, $\left.J=7.6 \mathrm{~Hz}, 2 \mathrm{H}, 8 \mathrm{a}-\mathrm{H}\right), 4.31$ (4.29) (d, $J=$ $8.6 \mathrm{~Hz}, 1 \mathrm{H}, 17-\mathrm{H}), 4.75(4.50)$ (q, $J=7.2 \mathrm{~Hz}, 1 \mathrm{H}, 18-\mathrm{H}$ ), $5.27\left(\mathrm{~d}, J=19.8 \mathrm{~Hz}, 1 \mathrm{H}, 13^{2}-\mathrm{H}\right), 5.12(\mathrm{~d}, J=19.8 \mathrm{~Hz}, 1 \mathrm{H}$, $\left.13^{2}-\mathrm{H}\right), 5.53 \sim 5.64$ (m, 1H, 5'-H), 6.91 (6.89) (6.88) (6.50) (t, $J=7.0 \mathrm{~Hz}, 1 \mathrm{H}, 3$ '-H), 8.59 (8.58), 8.95 (8.94), 9.48 (9.47) (each s, each $1 \mathrm{H}$, meso-H); IR $(\mathrm{KBr}) v: 3433(\mathrm{~N}-$ H), 2973 $2870(\mathrm{C}-\mathrm{H}), 1735 \sim 1695(\mathrm{C}=\mathrm{O}), 1605(\mathrm{C}=$ C), 1557 (chlorin skeleton), 1440, 1326, 1172, 1013, 809 $\mathrm{cm}^{-1}$; MS $m / z$ : $605.4(\mathrm{M}+\mathrm{H})^{+}$. Anal. calcd for $\mathrm{C}_{36} \mathrm{H}_{40} \mathrm{~N}_{6}$ $\mathrm{O}_{3}$ : C 71.50, H 6.67, N 13.90; found C 71.69, H 6.41, N 13.75 .

\subsection{3 -[1'( $R, S)$-1'-(2' $(R, S)$-甲基环丙基)]-3-去乙烯基焦 脱镁叶绿酸 $-a$ 甲酯 $(6)$ 的合成}

向 $10 \mathrm{~mL}$ 二苯醚中加入 $105 \mathrm{mg}$ 化合物 $\mathbf{5}(0.174$ $\mathrm{mmol})$, 于 $160{ }^{\circ} \mathrm{C}$ 油浴搅拌反应 $20 \mathrm{~min}$, 加入正已烷稀 释, 过滤除去大部分二苯醚, 以少量二氯甲烷溶解固体 混合物, 经柱层析纯化 [洗脱剂: $V$ (石油醚) $: V($ 乙酸乙 酯 $)=4: 1$ ], 得 $70 \mathrm{mg}$ 黑绿色固体 $6(0.122 \mathrm{mmol})$, 产率 为 $70 \%$. m.p. $215 \sim 217{ }^{\circ} \mathrm{C}$; UV-Vis $\left(\mathrm{CH}_{2} \mathrm{Cl}_{2}\right) \lambda_{\max }$ $\left[\varepsilon /\left(\mathrm{L} \cdot \mathrm{mol}^{-1} \cdot \mathrm{cm}^{-1}\right)\right]: 415\left(2.55 \times 10^{5}\right), 528\left(3.67 \times 10^{4}\right), 565$ $\left(3.38 \times 10^{4}\right), 599\left(4.16 \times 10^{4}\right), 659\left(2.06 \times 10^{4}\right) \mathrm{nm} ;{ }^{1} \mathrm{H}$ $\operatorname{NMR}\left(\mathrm{CDCl}_{3}\right) \delta$ : $-1.62(-1.69)(\mathrm{br}, 1 \mathrm{H}, \mathrm{NH}), 0.50$ (br, $1 \mathrm{H}, \mathrm{NH}), 1.66$ (t, $\left.J=7.6 \mathrm{~Hz}, 3 \mathrm{H}, 8 \mathrm{~b}-\mathrm{CH}_{3}\right), 1.77$ (d, $J=7.3$ $\left.\mathrm{Hz}, 3 \mathrm{H}, 18-\mathrm{CH}_{3}\right), 1.38 \sim 1.45(\mathrm{~m}, 1 \mathrm{H}, 3 \mathrm{~b}-\mathrm{H}), 1.51 \sim 1.65$ (m, 2H, 3b-H), $2.18 \sim 2.30\left(\mathrm{~m}, 3 \mathrm{H}, 17^{1}-\mathrm{H}+17^{2}-\mathrm{H}\right), 2.32 \sim$ $2.40(\mathrm{~m}, 1 \mathrm{H}, 17 \mathrm{a}-\mathrm{H}+17 \mathrm{~b}-\mathrm{H}), 2.46 \sim 2.70(\mathrm{~m}, 2 \mathrm{H}, 17 \mathrm{a}-\mathrm{H}+$ 17b-H+3a-H), 3.21 (3.20), 3.34 (3.38), 3.60, 3.61 (each s, each $\left.3 \mathrm{H}, \mathrm{CH}_{3}+\mathrm{OCH}_{3}\right), 3.62(\mathrm{q}, J=7.6 \mathrm{~Hz}, 2 \mathrm{H}, 8 \mathrm{a}-\mathrm{H})$, $4.23(\mathrm{~d}, J=8.0 \mathrm{~Hz}, 1 \mathrm{H}, 17-\mathrm{H}), 4.41$ (q, $J=7.3 \mathrm{~Hz}, 1 \mathrm{H}$, $18-\mathrm{H}), 5.20$ (d, $\left.J=19.8 \mathrm{~Hz}, 1 \mathrm{H}, 13^{2}-\mathrm{H}\right), 5.05$ (d, $J=19.8$ $\left.\mathrm{Hz}, 1 \mathrm{H}, 13^{2}-\mathrm{H}\right), 8.42$ (8.39), 8.38 (8.34), 9.41 (9.39) (each $\mathrm{s}$, each $1 \mathrm{H}$, meso-H); IR $(\mathrm{KBr}) v: 3432(\mathrm{~N}-\mathrm{H}), 2800(\mathrm{C}-$ H), 1735 1689 $(\mathrm{C}=\mathrm{O}), 1654,1618(\mathrm{C}=\mathrm{C}), 1560$ (chlorin skeleton), 1400, 1251, 1163, $1082 \mathrm{~cm}^{-1}$; MS m/z: 577.4 $(\mathrm{M}+\mathrm{H})^{+}$. Anal. calcd for $\mathrm{C}_{36} \mathrm{H}_{40} \mathrm{~N}_{4} \mathrm{O}_{3}: \mathrm{C} 74.97, \mathrm{H} 6.99, \mathrm{~N}$ 9.71; found C 74.69, H 7.17, N 9.69. 
3.63 -[1' $(R, S)$-(1'-羟基-1'-环丙乙基)]-13'-(1.4-二氧环 戊基)-3-去乙烯基焦脱镁叶绿酸-a 甲酯(8)的合成

将 $260 \mathrm{mg}$ 化合物 7 (0.427 mmol) 溶解于 $20 \mathrm{~mL}$ 干燥 的四氢呋喃中, 在氮气保护中于 $0{ }^{\circ} \mathrm{C}$ 搅拌下缓慢滴加 1 $\mathrm{mL}$ 的 $1 \mathrm{~mol} / \mathrm{L}$ 环丙基澳化镁四氢呋喃溶液, 继续搅拌 $10 \mathrm{~min}$, 再升温至室温, 避光反应约 $1 \mathrm{~h}$ 后反应结束; 向 反应体系加入 $25 \mathrm{~mL}$ 的饱和氯化铵溶液, 搅拌 $15 \mathrm{~min}$, 再加入 $20 \mathrm{~mL}$ 的二氯甲烷, 分出有机层, 用二氯甲烷萃 取水相 $(15 \mathrm{~mL} \times 3)$, 合并有机层并减压浓缩, 将所得剩 余物经柱层析纯化 [洗脱剂: $V$ (石油醚) $: V$ (乙酸乙酯 $)=$ $3: 1]$, 得 $156 \mathrm{mg}$ 黑绿色固体 $8(0.239 \mathrm{mmol})$, 产率为 56\%. m.p. $224 \sim 227{ }^{\circ} \mathrm{C}$; UV-Vis $\left(\mathrm{CH}_{2} \mathrm{Cl}_{2}\right) \lambda_{\max }[\varepsilon /(\mathrm{L} \bullet$ $\left.\left.\mathrm{mol}^{-1} \cdot \mathrm{cm}^{-1}\right)\right]: 413\left(8.98 \times 10^{4}\right), 497\left(1.62 \times 10^{4}\right), 524$ $\left(8.08 \times 10^{3}\right), \quad 593 \quad\left(8.97 \times 10^{3}\right), \quad 610 \quad\left(8.08 \times 10^{3}\right), \quad 646$ $\left(4.49 \times 10^{4}\right) \mathrm{nm} ;{ }^{1} \mathrm{H}$ NMR $\left(\mathrm{CDCl}_{3}\right) \delta:-3.18(\mathrm{br}, 1 \mathrm{H}$, $\mathrm{NH}),-1.38(\mathrm{br}, 1 \mathrm{H}, \mathrm{NH}), 1.65(\mathrm{t}, J=7.6 \mathrm{~Hz}, 3 \mathrm{H}$, $\left.8 \mathrm{~b}-\mathrm{CH}_{3}\right), 1.75\left(\mathrm{~d}, J=7.0 \mathrm{~Hz}, 3 \mathrm{H}, 18-\mathrm{CH}_{3}\right), 1.46 \sim 1.60(\mathrm{~m}$, $4 \mathrm{H}$, cyclopropyl-2'-H), $1.90 \sim 2.40(\mathrm{~m}, 3 \mathrm{H}, 17 \mathrm{a}+17 \mathrm{~b}-\mathrm{H})$, $2.35\left(\mathrm{~s}, 3 \mathrm{H}, 3 \mathrm{a}-\mathrm{CH}_{3}\right), 2.50 \sim 2.78(\mathrm{~m}, 2 \mathrm{H}, 17 \mathrm{a}+17 \mathrm{~b}-\mathrm{H}+$ cyclopropyl-1'-H), 3.33, 3.52, 3.55, 3.56 (each s, each $3 \mathrm{H}$, $\mathrm{CH}_{3}+\mathrm{OCH}_{3}$ ), $3.68(\mathrm{q}, J=7.6 \mathrm{~Hz}, 2 \mathrm{H}, 8 \mathrm{a}-\mathrm{H}), 4.20 \sim 4.67$ $\left(\mathrm{m}, 6 \mathrm{H}, 17-\mathrm{H}+18 \mathrm{H}+13^{1}-\mathrm{OCH}_{2} \mathrm{CH}_{2} \mathrm{O}\right), 4.94(\mathrm{~d}, J=19.0$ $\left.\mathrm{Hz}, 1 \mathrm{H}, 13^{2}-\mathrm{H}\right), 5.09$ (d, $\left.J=19.0 \mathrm{~Hz}, 1 \mathrm{H}, 13^{2}-\mathrm{H}\right), 8.88$, 9.59, 10.57 (each s, each 1H, meso-H); IR (KBr) v: 3409 $(\mathrm{N}-\mathrm{H}), 2960(\mathrm{C}-\mathrm{H}), 1726 \sim 1701(\mathrm{C}=\mathrm{O}), 1647(\mathrm{C}=\mathrm{C})$, 1550 (chlorin skeleton), 1460, 1380, 1201, 1143, 1070 $\mathrm{cm}^{-1}$; MS m/z: $651.4(\mathrm{M}+\mathrm{H})^{+}$. Anal. calcd for $\mathrm{C}_{39} \mathrm{H}_{46} \mathrm{~N}_{4^{-}}$ $\mathrm{O}_{5}$ : C 71.97, H 7.12, N 8.61; found C 71.79, H 7.30, N 8.53 .

\subsection{3a-环丙基焦脱镁叶绿酸-a 甲酯(9)的合}

在 $25 \mathrm{~mL}$ 苯中溶解 $123 \mathrm{mg}$ 化合物 $8(0.189 \mathrm{mmol})$, 再加入 $3 \mathrm{mg}$ 对甲苯磺酸, 于 $90{ }^{\circ} \mathrm{C}$ 下避光搅拌反应 1.5 $\mathrm{h}$, 先后向反应体系加 $25 \mathrm{~mL}$ 水和 $25 \mathrm{~mL}$ 二氯甲烷, 分出 有机层并用无水硫酸钠干燥, 减压除去溶剂, 所得剩余 物经柱层析纯化 [洗脱剂: $V$ (石油醚) $: V($ 乙酸乙酯 $)=$ $4: 1]$, 得 $71 \mathrm{mg}$ 黑绿色固体 $9(0.121 \mathrm{mmol})$, 产率为 64\%. m.p. $201 \sim 203{ }^{\circ} \mathrm{C}$; UV-Vis $\left(\mathrm{CH}_{2} \mathrm{Cl}_{2}\right) \lambda_{\max }[\varepsilon /(\mathrm{L} \bullet$ $\left.\left.\mathrm{mol}^{-1} \cdot \mathrm{cm}^{-1}\right)\right]$ : $410\left(1.15 \times 10^{5}\right), 507\left(8.05 \times 10^{3}\right), 539$ $\left(6.90 \times 10^{3}\right), 607\left(2.53 \times 10^{4}\right), 661\left(5.52 \times 10^{4}\right) \mathrm{nm} ;{ }^{1} \mathrm{H}$ $\operatorname{NMR}\left(\mathrm{CDCl}_{3}\right) \delta$ : $-1.68(\mathrm{br}, 1 \mathrm{H}, \mathrm{NH}), 0.45(\mathrm{br}, 1 \mathrm{H}, \mathrm{NH})$, $1.48 \sim 1.62(\mathrm{~m}, 4 \mathrm{H}, 3 \mathrm{a}-(2 '-\mathrm{H})), 1.68$ (t, $J=7.6 \mathrm{~Hz}, 3 \mathrm{H}$, $\left.8 \mathrm{~b}-\mathrm{CH}_{3}\right), 1.77\left(\mathrm{~d}, J=7.0 \mathrm{~Hz}, 3 \mathrm{H}, 18-\mathrm{CH}_{3}\right), 2.15 \sim 2.38(\mathrm{~m}$, $2 \mathrm{H}, 17 \mathrm{a}-\mathrm{H}+17 \mathrm{~b}-\mathrm{H}), 2.43 \sim 2.64(\mathrm{~m}, 2 \mathrm{H}, 17 \mathrm{a}-\mathrm{H}+17 \mathrm{~b}-\mathrm{H})$, $2.70 \sim 2.82\left(\mathrm{~m}, 1 \mathrm{H}, 3 \mathrm{a}-\left(1^{\prime}-\mathrm{H}\right)\right), 3.19,3.26,3.58,3.69$ (each s, each $\left.3 \mathrm{H}, \mathrm{CH}_{3}+\mathrm{OCH}_{3}\right), 3.67$ (q, $\left.J=7.6 \mathrm{~Hz}, 2 \mathrm{H}, 8 \mathrm{a}-\mathrm{H}\right)$, $4.27(\mathrm{~d}, J=7.4 \mathrm{~Hz}, 1 \mathrm{H}, 17-\mathrm{H}), 4.44(\mathrm{q}, J=7.2 \mathrm{~Hz}, 1 \mathrm{H}$, $18-\mathrm{H}), 5.13$ (d, $\left.J=19.0 \mathrm{~Hz}, 1 \mathrm{H}, 13^{2}-\mathrm{H}\right), 5.25$ (d, $J=20.0$ $\left.\mathrm{Hz}, 1 \mathrm{H}, 13^{2}-\mathrm{H}\right), 5.53$ (s, 1H, 3b-H), $5.93(\mathrm{~s}, 1 \mathrm{H}, 3 \mathrm{~b}-\mathrm{H})$, 8.49, 9.17, 9.48 (each s, each 1H, meso-H); IR (KBr) v: $3323(\mathrm{~N}-\mathrm{H}), 2925(\mathrm{C}-\mathrm{H}), 1739 \sim 1687(\mathrm{C}=\mathrm{O}), 1602$ $(\mathrm{C}=\mathrm{C}), 1500$ (chlorin skeleton), 1450, 1263, 1089, 1041, $902,700 \mathrm{~cm}^{-1}$; MS m/z: $589.4(\mathrm{M}+\mathrm{H})^{+}$. Anal. calcd for $\mathrm{C}_{37} \mathrm{H}_{40} \mathrm{~N}_{4} \mathrm{O}_{3}$ : C 75.48, H 6.85, N 9.52; found C 75.61, H 6.80, N 9.31 .

\section{8 螺[环丙基- $1^{\prime}, 13^{2}$-焦脱镁叶绿酸-a 甲酯](10)的合 成}

将 $223 \mathrm{mg}$ 化合物 4 (0.406 mmol)、 $40 \mathrm{mg} N$-甲基甘 氨酸 $(0.449 \mathrm{mmol}$ )和 $40 \mathrm{mg}$ 多聚甲醛溶解于 $40 \mathrm{~mL}$ 甲苯 中, 氮气保护下回流反应 $6 \mathrm{~h}$, 减压除去甲苯, 所得剩余 物经柱层析纯化 [洗脱剂: $V$ (石油醚) : $V$ (乙酸乙酯 $)=$ $3: 1]$, 得 $26 \mathrm{mg}$ 黑绿色固体 $\mathbf{1 0}(0.045 \mathrm{mmol})$, 产率为 $11 \%$. m.p. $207 \sim 210{ }^{\circ} \mathrm{C}$; UV-Vis $\left(\mathrm{CH}_{2} \mathrm{Cl}_{2}\right) \lambda_{\max }[\varepsilon /(\mathrm{L} \cdot$ $\left.\left.\mathrm{mol}^{-1} \cdot \mathrm{cm}^{-1}\right)\right]: 414\left(1.52 \times 10^{5}\right), 516\left(1.37 \times 10^{4}\right), 548$ $\left(2.02 \times 10^{4}\right), 612\left(9.12 \times 10^{3}\right), 670\left(7.14 \times 10^{4}\right) \mathrm{nm} ;{ }^{1} \mathrm{H}$ $\operatorname{NMR}\left(\mathrm{CDCl}_{3}\right) \delta:-1.28(\mathrm{br}, 1 \mathrm{H}, \mathrm{NH}), 0.62(\mathrm{br}, 1 \mathrm{H}, \mathrm{NH})$, $1.70\left(\mathrm{t}, J=7.6 \mathrm{~Hz}, 3 \mathrm{H}, 8 \mathrm{~b}-\mathrm{CH}_{3}\right), 1.68(\mathrm{~d}, J=7.0 \mathrm{~Hz}, 3 \mathrm{H}$, $\left.18-\mathrm{CH}_{3}\right), 1.77 \sim 1.96\left(\mathrm{~m}, 4 \mathrm{H}, 13^{2}-\mathrm{CH}_{2} \mathrm{CH}_{2}\right), 2.05 \sim 2.13$ $(\mathrm{m}, \quad 1 \mathrm{H}, \quad 17 \mathrm{a}-\mathrm{H}+17 \mathrm{~b}-\mathrm{H}), 2.26 \sim 2.44(\mathrm{~m}, 2 \mathrm{H}, \quad 17 \mathrm{a}+$ 17b-H), $2.53 \sim 2.60$ (m, 1H, 17a +17b-H), 3.22, 3.38, 3.63, 3.67 (each s, each $3 \mathrm{H}, \mathrm{CH}_{3}+\mathrm{OCH}_{3}$ ), $3.68(\mathrm{q}, J=7.6 \mathrm{~Hz}$, $2 \mathrm{H}, 8 \mathrm{a}-\mathrm{H}), 3.82$ (dd, $J=9.8,1.2 \mathrm{~Hz}, 1 \mathrm{H}, 17-\mathrm{H}), 4.34$ (q, $J=7.3 \mathrm{~Hz}, 1 \mathrm{H}, 18-\mathrm{H}), 6.34(\mathrm{dd}, J=17.8,1.3 \mathrm{~Hz}, 1 \mathrm{H}$, trans-3b-H), 6.16 (dd, $J=11.5,1.3 \mathrm{~Hz}, 1 \mathrm{H}$, cis-3b-H), 7.98 (dd, $J=17.8,11.5 \mathrm{~Hz}, 1 \mathrm{H}, 3 \mathrm{a}-\mathrm{H}$ ), 8.48, 9.30, 9.48 (each s, each $1 \mathrm{H}$, meso-H); IR $(\mathrm{KBr}) v: 3454(\mathrm{~N}-\mathrm{H}), 2858(\mathrm{C}-$ $\mathrm{H}), 1708 \sim 1787(\mathrm{C}=\mathrm{O}), 1618(\mathrm{C}=\mathrm{C}), 1461$ (chlorin skeleton), 1448, 1380, 1253, 1172, 1083, 1010, $801 \mathrm{~cm}^{-1}$; MS $m / z$ : $575.4(\mathrm{M}+\mathrm{H})^{+}$. Anal. calcd for $\mathrm{C}_{36} \mathrm{H}_{38} \mathrm{~N}_{4} \mathrm{O}_{3}: \mathrm{C}$ 75.24, H 6.66, N 9.75; found C 75.40, H 6.58, N 9.69.

\subsection{3-环丙基-3-去乙烯基焦脱镁叶绿酸-a 甲酯(11)的 合成}

在 $5 \mathrm{~mL}$ 干燥的二苯醚中溶解 $188 \mathrm{mg}$ 化合物 4 (0.343 mmol), 然后加入 $25 \mathrm{~mL}$ 重氮甲烷乙醚溶液, 塞 紧反应瓶口, 室温避光条件下放置 $24 \mathrm{~h}$, 常压下将未反 应的重氮甲烷蒸出回收, 剩余物在 $160{ }^{\circ} \mathrm{C}$ 下搅拌反应 $1.5 \mathrm{~h}$, 加入正己烷稀释, 过滤除去二苯醚, 浓缩后经硅 胶柱层析分离[洗脱剂: $V$ (石油醚) $: V($ 乙酸乙酯 $)=4$ : $1]$, 得 $129 \mathrm{mg}$ 暗红色固体产物 $\mathbf{1 1}(0.230 \mathrm{mmol})$, 产率为 $67 \%$. 物理常数及分析数据与文献 $[12]$ 一致. 
3.10 3-环丙基-20-氯-3-去乙烯基焦脱镁叶绿酸-a 甲 酯(12)的合成

将 $68 \mathrm{mg}$ 二氢卟吩 $11(0.121 \mathrm{mmol})$ 溶解于 $30 \mathrm{~mL}$ 四氢呋喃中, 室温条件下缓慢滴加由 $2 \mathrm{~mL} 30 \%$ 过氧化 氢和 $30 \mathrm{~mL}$ 稀盐酸 $(0.5 \mathrm{~mol} / \mathrm{L})$ 组成的混合液, $80{ }^{\circ} \mathrm{C}$ 回流 反应 $1 \mathrm{~h}$, 搅拌下向反应体系加入 $80 \mathrm{~mL}$ 水, 分出有机 层, 用二氯甲烷萃取水相 $(25 \mathrm{~mL} \times 3)$; 合并有机相, 先 后用 $10 \%$ 碳酸氢钠和冷水洗涤, 无水硫酸钠干燥, 减压 除去溶剂, 柱层析分离剩余物[洗脱剂: $V$ (石油醚)： $V$ (乙酸乙酯 $)=5: 2]$, 得 $53 \mathrm{mg}$ 暗红色固体 12 (0.089 $\mathrm{mmol}$ ), 产率为 $74 \%$. m.p. $235 \sim 237{ }^{\circ} \mathrm{C}$; UV-Vis $\left(\mathrm{CH}_{2} \mathrm{Cl}_{2}\right) \lambda_{\max }\left[\varepsilon /\left(\mathrm{L} \cdot \mathrm{mol}^{-1} \cdot \mathrm{cm}^{-1}\right)\right]: 411\left(1.65 \times 10^{5}\right), 508$ $\left(3.30 \times 10^{3}\right), \quad 536\left(4.95 \times 10^{3}\right), \quad 610 \quad\left(4.95 \times 10^{3}\right), \quad 668$ $\left(6.27 \times 10^{4}\right) \mathrm{nm} ;{ }^{1} \mathrm{H}$ NMR $\left(\mathrm{CDCl}_{3}\right) \delta:-1.97$ (br, $1 \mathrm{H}$, $\mathrm{NH}),-0.92(\mathrm{br}, 1 \mathrm{H}, \mathrm{NH}), 1.24 \sim 1.29(\mathrm{~m}, 2 \mathrm{H}, 3 \mathrm{~b}-\mathrm{H})$, $1.62 \sim 1.68(\mathrm{~m}, 2 \mathrm{H}, 3 \mathrm{~b}-\mathrm{H}), 1.61(\mathrm{~d}, J=7.2 \mathrm{~Hz}, 3 \mathrm{H}$, $\left.18-\mathrm{CH}_{3}\right), 1.67\left(\mathrm{t}, J=7.6 \mathrm{~Hz}, 3 \mathrm{H}, 8 \mathrm{~b}-\mathrm{CH}_{3}\right), 2.10 \sim 2.22(\mathrm{~m}$, $2 \mathrm{H}, 17 \mathrm{a}-\mathrm{H}+17 \mathrm{~b}-\mathrm{H}), 2.45 \sim 2.61(\mathrm{~m}, 2 \mathrm{H}, 17 \mathrm{a}-\mathrm{H}+17 \mathrm{~b}-\mathrm{H})$, $2.71 \sim 2.78(\mathrm{~m}, 1 \mathrm{H}, 3 \mathrm{a}-\mathrm{H}), 3.27,3.58,3.62,3.64$ (each s, each $\left.3 \mathrm{H}, \mathrm{CH}_{3}+\mathrm{OCH}_{3}\right), 3.67$ (q, $\left.J=7.6 \mathrm{~Hz}, 2 \mathrm{H}, 8 \mathrm{a}-\mathrm{H}\right)$, $4.20(\mathrm{dd}, J=8.5,2.8 \mathrm{~Hz}, 1 \mathrm{H}, 17-\mathrm{H}), 4.77$ (q, $J=7.1 \mathrm{~Hz}$, $1 \mathrm{H}, 18-\mathrm{H}), 5.18$ (d, $\left.J=19.8 \mathrm{~Hz}, 1 \mathrm{H}, 13^{2}-\mathrm{H}\right), 5.24$ (d, $J=$ $19.8 \mathrm{~Hz}, 1 \mathrm{H}, 13^{2}-\mathrm{H}$ ), 9.47, 9.86 (each s, each $1 \mathrm{H}$, meso-H); IR $(\mathrm{KBr}) v: 3436(\mathrm{~N}-\mathrm{H}), 2925(\mathrm{C}-\mathrm{H}), 1704 \sim 1788$ $(\mathrm{C}=\mathrm{O}), 1674(\mathrm{C}=\mathrm{C}), 1539$ (chlorin skeleton), 1519, 1400, 1176, 1054, 906, $730 \mathrm{~cm}^{-1}$; MS m/z: $598.2(\mathrm{M}+2)^{+}$. Anal. calcd for $\mathrm{C}_{35} \mathrm{H}_{37} \mathrm{ClN}_{4} \mathrm{O}_{3}$ : C 70.40, H 6.25, N 9.38; found $\mathrm{C} 70.60, \mathrm{H} 6.41, \mathrm{~N} 9.31$.

\subsection{3 -环丙基- $13^{2}$-氧代-3-去乙烯基焦脱镁叶绿酸-a 甲酯(13)的合成}

在开放的容器中, 将 $187 \mathrm{mg}$ 化合物 11 (0.332 $\mathrm{mmol}$ )溶于 $15 \mathrm{~mL}$ 四氢呋喃中, 再加入 $0.425 \mathrm{~g} \mathrm{LiOH}($ 溶 解于 $4 \mathrm{~mL}$ 水中, 用约 $8 \mathrm{~mL} \mathrm{MeOH}$ 稀释), 室温避光剧烈 搅拌 $6 \mathrm{~h}$, 搅拌下用 $\mathrm{AcOH}$ 调至 $\mathrm{pH}$ 为 $2 \sim 3$, 加入 $100 \mathrm{~mL}$ 氯仿和 $150 \mathrm{~mL}$ 水分层, 水相用二氯甲烷萃取(15 $\mathrm{mL} \times 3)$, 合并有机层, 水洗至水层为中性, 无水硫酸钠 干燥, 减压蒸干. 用约 $5 \mathrm{~mL}$ 二氯甲烷溶解, 加入略超计 算量的重氮甲烷, 振摇后迅速用乙酸淬灭反应, 减压除 尽溶剂, 柱层析分离剩余物 [洗脱剂: $V$ (石油醚) : $V$ (乙 酸乙酯 $)=3: 1]$, 得 $44 \mathrm{mg}$ 黄色固体 13 (0.076 mmol), 产率为 $23 \%$. m.p. $227 \sim 230{ }^{\circ} \mathrm{C}$; UV-Vis $\left(\mathrm{CH}_{2} \mathrm{Cl}_{2}\right) \lambda_{\text {max }}$ $\left[\varepsilon /\left(\mathrm{L} \cdot \mathrm{mol}^{-1} \cdot \mathrm{cm}^{-1}\right)\right]: 410\left(1.21 \times 0^{5}\right), 507\left(8.47 \times 10^{3}\right), 535$ $\left(1.45 \times 10^{4}\right), \quad 610 \quad\left(3.99 \times 10^{4}\right), \quad 643 \quad\left(2.78 \times 10^{4}\right) \quad 670$ $\left(4.11 \times 10^{4}\right) \mathrm{nm} ;{ }^{1} \mathrm{H}$ NMR $\left(\mathrm{CDCl}_{3}\right) \delta:-2.39$ (br, $1 \mathrm{H}$, $\mathrm{NH}),-0.09$ (br, 1H, NH), $1.24 \sim 1.36(\mathrm{~m}, 2 \mathrm{H}, 3 \mathrm{~b}-\mathrm{H})$,
$1.52 \sim 1.68(\mathrm{~m}, 2 \mathrm{H}, 3 \mathrm{~b}-\mathrm{H}), 1.76(\mathrm{t}, J=7.6 \mathrm{~Hz}, 3 \mathrm{H}$, $\left.8 \mathrm{~b}-\mathrm{CH}_{3}\right), 1.84\left(\mathrm{~d}, J=7.4 \mathrm{~Hz}, 3 \mathrm{H}, 18-\mathrm{CH}_{3}\right), 1.94 \sim 2.09(\mathrm{~m}$, $1 \mathrm{H}, 17 \mathrm{a}-\mathrm{H}+17 \mathrm{~b}-\mathrm{H}), 2.26 \sim 2.38(\mathrm{~m}, 2 \mathrm{H}, 17 \mathrm{a}-\mathrm{H}+17 \mathrm{~b}-\mathrm{H})$, $2.62 \sim 2.83(\mathrm{~m}, 2 \mathrm{H}, 17 \mathrm{a}-\mathrm{H}+17 \mathrm{~b}-\mathrm{H}+3 \mathrm{a}-\mathrm{H}), 3.18,3.39$, $3.58,3.88$ (each s, each $3 \mathrm{H}, \mathrm{CH}_{3}+\mathrm{OCH}_{3}$ ), 3.82 (q, $J=7.6$ $\mathrm{Hz}, 2 \mathrm{H}, 8 \mathrm{a}-\mathrm{H}), 4.66$ (q, $J=7.2 \mathrm{~Hz}, 1 \mathrm{H}, 18-\mathrm{H}), 5.17$ (d, $J=$ $9.3 \mathrm{~Hz}, 1 \mathrm{H}, 17-\mathrm{H}$ ), 8.91, 0.90, 10.09 (each s, each 1H, meso-H); IR (KBr) v: $3449(\mathrm{~N}-\mathrm{H}), 2924(\mathrm{C}-\mathrm{H}), 1742$ $1686(\mathrm{C}=\mathrm{O}), 1637(\mathrm{C}=\mathrm{C}), 1560$ (chlorin skeleton), 1459, 1341, 1086, 669, $536 \mathrm{~cm}^{-1}$; MS m/z: $577.4(\mathrm{M}+\mathrm{H})^{+}$. Anal. calcd for $\mathrm{C}_{35} \mathrm{H}_{36} \mathrm{~N}_{4} \mathrm{O}_{4}$ : C 72.90, H 6.29, N 9.72; found C 72.79, H 6.50, N 9.69.

\subsection{3-环丙基-3-去乙烯基红紫素-18 甲酯(14)的合成}

将 $78 \mathrm{mg}$ 化合物 $\mathbf{1 3}(0.135 \mathrm{mmol})$ 溶于 $15 \mathrm{~mL}$ 四氢呋 喃中, 加入 $5 \mathrm{~mL}$ 含有 $25 \mathrm{mg}$ 甲醇钠的甲醇溶液, 室温避 光搅拌 $2 \mathrm{~h}$, 搅拌下用 $\mathrm{AcOH}$ 调至 $\mathrm{pH}$ 为 $2 \sim 3$, 加入 45 $\mathrm{mL}$ 二氯甲烷和 $50 \mathrm{~mL}$ 水分层, 水相用二氯甲烷萃取(15 $\mathrm{mL} \times 3)$, 合并有机层, 水洗三次, 无水硫酸钠干燥, 浓 缩至 $5 \mathrm{~mL}$ 体积后加入重氮甲烷甲基化，振摇后迅速用 乙酸淬灭反应, 减压除尽溶剂, 柱层析分离剩余物 [洗 脱剂: $V$ (石油醚) $: V($ 乙酸乙酯 $)=4: 1$ ], 得 $64 \mathrm{mg}$ 黄色 固体 14 (0.108 mmol), 产率为 $80 \%$. 物理常数及分析数 据与文献 $[13]$ 一致.

\subsection{N-环丙甲基-3-环丙基-3-红紫素-18 亚酰胺甲酯 (15)的合成}

将 $120 \mathrm{mg}$ 红紫素-18 (14) (0.203 mmol)溶解于 $5 \mathrm{~mL}$ 甲苯中, 加入 2 滴环丙甲基胺, $120{ }^{\circ} \mathrm{C}$ 回流搅拌反应, TLC 检测反应进程, $3 \mathrm{~h}$ 反应完毕, 减压蒸除甲苯, 柱层 析分离剩余物 [洗脱剂: $V$ (石油醚) : $V$ (乙酸乙酯 $)=3$ : $1]$, 得 $80 \mathrm{mg}$ 红色固体 15 (0.124 mmol), 产率为 $61 \%$. m.p. 235 $237{ }^{\circ} \mathrm{C}$; UV-Vis $\left(\mathrm{CH}_{2} \mathrm{Cl}_{2}\right) \lambda_{\max }\left[\varepsilon /\left(\mathrm{L} \cdot \mathrm{mol}^{-1}\right.\right.$ • $\left.\left.\mathrm{cm}^{-1}\right)\right]: \quad 413 \quad\left(1.43 \times 10^{5}\right), \quad 422 \quad\left(1.09 \times 10^{4}\right), \quad 508$ $\left(1.46 \times 10^{4}\right), 544\left(3.40 \times 10^{4}\right), 695\left(0.527 \times 10^{4}\right) \mathrm{nm} ;{ }^{1} \mathrm{H}$ NMR $\left(\mathrm{CDCl}_{3}\right) \delta$ : -0.25 (br, $\left.1 \mathrm{H}, \mathrm{NH}\right),-0.03$ (br, $1 \mathrm{H}$, $\mathrm{NH}), 1.67$ (t, $\left.J=7.6 \mathrm{~Hz}, 3 \mathrm{H}, 8 \mathrm{~b}-\mathrm{CH}_{3}\right), 1.75$ (d, $J=7.3 \mathrm{~Hz}$, $\left.3 \mathrm{H}, 18-\mathrm{CH}_{3}\right), 1.22 \sim 1.34\left(\mathrm{~m}, 2 \mathrm{H}\right.$, cyclopropyl- $\left.\mathrm{CH}_{2} \mathrm{CH}_{2}\right)$, $1.37 \sim 1.48\left(\mathrm{~m}, 2 \mathrm{H}\right.$, cyclopropyl- $\left.\mathrm{CH}_{2} \mathrm{CH}_{2}\right), 1.53 \sim 1.75(\mathrm{~m}$, $2 \mathrm{H}$, cyclopropyl- $\left.\mathrm{CH}_{2} \mathrm{CH}_{2}\right), 1.92 \sim 2.05(\mathrm{~m}, 3 \mathrm{H}$, cyclopropyl- $\left.\mathrm{CH}_{2} \mathrm{CH}_{2}\right), 2.28 \sim 2.45(\mathrm{~m}, 2 \mathrm{H}, 17 \mathrm{a}-\mathrm{H}+17 \mathrm{~b}-\mathrm{H}), 2.64 \sim$ $2.78(\mathrm{~m}, 3 \mathrm{H}, 17 \mathrm{a}-\mathrm{H}+17 \mathrm{~b}-\mathrm{H}+3 \mathrm{a}-\mathrm{H}), 3.20,3.32,3.56,3.83$ (each s, each $3 \mathrm{H}, \mathrm{CH}_{3}+\mathrm{OCH}_{3}$ ), 3.66 (q, $J=7.6 \mathrm{~Hz}, 2 \mathrm{H}$, $8 \mathrm{a}-\mathrm{H}), 4.32$ (q, $J=7.0 \mathrm{~Hz}, 1 \mathrm{H}, 18-\mathrm{H}), 5.37$ (d, $J=7.6 \mathrm{~Hz}$, $1 \mathrm{H}, 17-\mathrm{H}), 8.49,9.53,9.61$ (each s, each $1 \mathrm{H}$, meso-H); IR $(\mathrm{KBr}) v: 3433 \quad(\mathrm{~N}-\mathrm{H}), 2973 \sim 2870(\mathrm{C}-\mathrm{H}), 1735 \sim$ $1695(\mathrm{C}=\mathrm{O}), 1605(\mathrm{C}=\mathrm{C}), 1557$ (chlorin skeleton), 1440, 
1326, 1172, 1013, $809 \mathrm{~cm}^{-1}$; MS m/z: $646.4(\mathrm{M}+\mathrm{H})^{+}$. Anal. calcd for $\mathrm{C}_{39} \mathrm{H}_{43} \mathrm{~N}_{5} \mathrm{O}_{4}: \mathrm{C}$ 72.53, $\mathrm{H}$ 6.71, N 10.84; found C 72.71, H 6.58, N 10.97.

\subsection{4 体外光动力药理活性的测定}

待测样品和对照品：分别选择化合物 $2,3 a, 3 b, 4$, 11,12 和 15 为待测化合物, 选择临床光动力抗癌药物光 敏素 (Photofrin) 和中位 meso- 四羟苯基二氢卟吩 (mTHPC) 为对照品. 将待测样品和对照品用含 $20 \%$ 增溶 剂聚氧乙烯蓖麻油的水溶液溶解.

测定方法(MTT 比色检测法): 在 $37{ }^{\circ} \mathrm{C}, 5 \% \mathrm{CO}_{2}$, 饱 和湿度下, 小鼠肉瘤 $\mathrm{S}-180$ 细胞贴壁培养于含 $10 \mathrm{~mL}$ 完 全培养液 $(\alpha-\mathrm{MEM}$, 含 $10 \%$ 新生牛血清, $L$-谷氨酸盐, 青 需素以及链霉素) 的细胞培养瓶中. 细胞接种于含完全 培养基的 96 孔细胞培养板中, 每孔细胞密度为 $5 \times 10^{3}$ 个/孔. $37{ }^{\circ} \mathrm{C}$ 恒温培养过夜后各孔分别加入不同浓度的 光敏剂, 使其最终浓度分别为 $10,1,0.1,0.05,0.01$ $\mu \mathrm{mol} / \mathrm{L}$, 在暗处 $37{ }^{\circ} \mathrm{C}$ 继续恒温培养 $24 \mathrm{~h}$. 在光处理前用 不含药物的完全培养基替换孔中的培养基, 然后用 670 700 $\mathrm{nm}$ 的光照射细胞 $20 \mathrm{~min}$, 总光剂量为 $8.4 \mathrm{~J}$. 细胞在暗处于 $37{ }^{\circ} \mathrm{C}$ 继续恒温培养 $48 \mathrm{~h}$. 在培养结束前 4 $\mathrm{h}$, 分别在每孔中加入 $10 \mu \mathrm{L} 4.0 \mathrm{mg} / \mathrm{mL}$ 的 3-[2'-(4',5'-二 甲基噻唑)]-2,5-二苯基四唑溴化物(MTT)磷酸缓冲溶液. $4 \mathrm{~h}$ 后移除培养基和 MTT, 加入 $100 \mu \mathrm{L} \mathrm{DMSO}$ 溶解甲暨 结晶. 用酶标仪(Titertek Multiscan Plus MK II; Miles Inc.) 测定各孔在 $560 \mathrm{~nm}$ 的吸光度. 每组实验重复 3 次, 标准误差(SEMs) 小于 $10 \%$, 每次实验设 5 个重复孔. 以 不加光敏剂和非光照条件下的细胞为空白对照, 以光敏 剂 3 次重复实验所得吸光度平均值对光敏剂浓度作图得 到各光敏剂的 $\mathrm{IC}_{50}$ 值. 结果分析及各化合物的 $\mathrm{IC}_{50}$ 值见 表 1 .

\section{References}

[1] (a) Chen, Y. H.; Li, G. L.; Pandey, R. K. Curr. Org. Chem. 2004, 8, 1105.

(b) Gryshuk, A. L.; Chen, Y.-H.; Potter, W.; Ohulchanskyy, T.; Oseroff, A.; Pandey, P. K. J. Med. Chem. 2006, 49, 1874.

(c) Gil, M.; Bieniaszl, M.; Seshadri, M.; Fisher, D.; Ciesielski, M. J.; Chen, Y.; Pandey, R. K.; Kozbor, D. Brit. J. Cancer 2011, 105, 1512.

[2] (a) Goswami, L. N.; Ethirajan, M.; Dobhal, M. P.; Zhang, M.; Missert, J. R.; Shibata, M.; Kadish, K. M.; Pandey, R. K. J. Org. Chem. 2009, 74, 568

(b) Ethirajan, M.; Joshi, P.; William, W. H.; Ohkubo, K.; Fukuzumi, S.; Pandey, R. K. Org. Lett. 2011, 13, 1956.
[3] (a) Pandey, P. K.; Zhang, X.; Morgan, J.; Missort, J. R.; Liu, T.-H.; Shibata, M.; Bellnier, D. A.; Oseroff, A. R.; Henderson, B. W.; Dougherty, T. J.; Pandey, P. K. Mol. Pharmaceutics 2007, 4, 448. (b) Bellnier, D. A.; Greco, W. R.; Loewen, G. M.; Nava, H.; Oseroff, A. R.; Pandey, R. K.; Tsuchida, T.; Dougherty, T. J. Cancer Res. 2003, 63, 1806;

[4] (a) Gil, M.; Bieniaszl, M.; Seshadri, M.; Fisher, D.; Ciesielski, M. J.; Chen, Y.; Pandey, R. K.; Kozbor, D. Brit. J. Cancer 2011, 103(10), 1.

(b) Bellnier, D. A.; Greco, W. R.; Loewen, G. M.; Nava, H.; Oseroff, A. R.; Pandey, R. K.; Tsuchida, T.; Dougherty, T. J. Cancer Res. 2003, 63, 1806.

[5] (a) Tamiaki, H.; Shibata, R.; Mizoguchi, T. Photochem. Photobiol. 2007, 83, 152 .

(b) Goswami, L. N.; Ethirajan, M.; Dobhal, M. P.; Zhang, M.; Missert, J. R.; Shibata, M.; Kadish, K. M.; Pandey, R. K. J. Org. Chem. 2009, 74, 568 .

(c) Hoober, J. K.; Eggink, L. L.; Chen, M. Photosynth. Res. 2007, 94, 387.

(d) Wang, J.-J. Chin. J. Org. Chem. 2005, 25, 1353 (in Chinese). (王进军, 有机化学, 2005, 25, 1353.)

[6] (a) Wang, J. J.; Li, J.-J.; Wu, X.-R.; Shim, Y.-K. Chin. J. Chem. 2006, 24, 933.

(b) Wang, J.-J.; Li, J.-Z.; Li, Y.-W.; Jakus, J.; Shim, Y.-K. J. Porphyrins Phthalocyanines 2010, 14, 859.

(c) Li, J.-Z.; Wang, J.-J.; Yoon, L.; Cui, B.-C.; Shim, Y.-K. Bioorg. Med. Chem. Lett. 2012, 22, 1846.

(d) Wang, J. J.; Liu, C.-L.; Li, J.-J. Synth. Commun. 2012, 42, 487.

(e) Wang, L.-M.; Wang, Z.; Yang. Z.; Jin, Y.-X.; Wang, J.-J. Chin. J. Org. Chem. 2012, 32, 2154 (in Chinese).

(王鲁敏, 王振, 杨泽, 金英学, 王进军, 有机化学, 2012, 32, 2154.)

(f) Wang, J.-J.; Li, F.-G.; Li, Y.-W. Chin. J. Org. Chem. 2011, 31, 68 (in Chinese).

(王进军, 李付国, 李韵伟, 有机化学, 2011, 31, 68.)

[7] Tamiaki, H.; Miyatake, T.; Tanikaga, R. Tetrahedron Lett. 1997, $38,267$.

[8] Li, J.-Z. M. S. Thesis, Yantai University, Yantai, 2007 (in Chinese). (李家柱, 硕士论文, 烟台大学, 烟台, 2007.)

[9] Li, J.-Z. M. S. Thesis, Yantai University, Yantai, 2010 (in Chinese). (张朋, 硕士论文, 烟台大学, 烟台, 2010.)

[10] Smith, K. M.; Gogg, D. A.; Simpson, D. J. J. Am. Chem. Soc. 1985, 107,4946

[11] Wang, J.-J.; Zhao, Y.; Wu, X.-R.; Han, G.-F.; Shin, R.-K. Chin. J. Org. Chem. 2002, 22, 565 (in Chinese).

(王进军, 赵岩, 邬旭然, 韩光范, 沈荣基, 有机化学, 2002, 22, 565.)

[12] Ji, J.-Y.; Li, J.-Z.; Wang, H.; Li, F.-G.; Han, G.-F.; Shen, R.-K.; Wang, J.-J. Chin. J. Org. Chem. 2006, 26, 1714 (in Chinese). (纪建业, 李家柱, 王虎, 李付国, 韩光范, 沈荣基, 王进军, 有 机化学, 2006, 26, 1714.)

[13] Liu, R.-R.; Yin, J.-G.; Li, J. Z.; Wu, J.; Chen, G.-L.; Jin, Y.-X.; Wang, J.-J. Chin. J. Org. Chem. 2012, 32. 544 (in Chinese). (刘再冉, 殷军港, 李家柱, 武进, 金英学, 王进军, 有机化学, 2012, 32, 544.) 